\title{
Behavior for Time Invariant Finite Dimensional Discrete Linear Systems
}

\author{
Sisilia Sylviani, Hanni Garminia \& Pudji Astuti \\ Algebra Research Group, Faculty of Mathematics and Natural Sciences \\ Institut Teknologi Bandung, Jalan Ganesa 10, Bandung 40132, Indonesia \\ Email: pudji@math.itb.ac.id
}

\begin{abstract}
The behavior of a dynamical system, in Willems's point of view, is the set of all trajectories of the system. Fuhrmann defines a behavior as a linear, shift invariant, and complete subspace of $z^{-1} \mathbb{F}^{m}\left[\left[z^{-1}\right]\right]$, the vector space consisting of power series in $z^{-1}$ with coefficients in signal space $\mathbb{W}=\mathbb{F}^{m}$. In this paper we show that the behavior of a finite dimensional, time invariant discrete linear system in Willems's setting is also a behavior according to Fuhrmann's.
\end{abstract}

Keywords: behavior; complete; linear system; shift invariant; trajectories.

\section{$1 \quad$ Introduction}

Behavioral theory is a mathematical idea to study dynamical systems through the set of trajectories of the systems. The basic concept of the behavioral framework deals with a mathematical model of a system for what a model ought to be [1], highlighting some physical properties and practical questions of the system. Willems [1,2] defines a dynamical system as a triple, $\sum=(\mathbb{T}, \mathbb{W}, \mathfrak{B})$, where $\mathbb{T} \subseteq \mathbb{R}$ is the time axis, $\mathbb{W}$ is called the signal space, $\mathbb{W}^{\mathbb{T}}$ is the set of all transformations from $\mathbb{T}$ to $\mathbb{W}$, and $\mathfrak{B} \subseteq \mathbb{W}^{\mathbb{T}}$ is called behavior. The elements of $\mathfrak{B}$ are called the trajectories of the system. This definition is very general and can be loosely described.

In general, the behavior of a linear system, in Willems's point of view [2] represents the set of all the trajectories of the system. Meanwhile, Fuhrmann [3] adopts Willems's [2] concept of a behavior for a discrete linear system by restricting $\mathbb{T}$ to the set of natural numbers, denoted by $\mathbb{Z}_{+}$, and $\mathbb{W}$ to $\mathbb{F}^{m}$, the $m$ dimensional vector space over a field $\mathbb{F}$. However, Fuhrmann does not define the behavior of a time invariant discrete linear system as the set of all the system trajectories. Fuhrmann defines a behavior as a linear, shift invariant, and complete subspace of the set $z^{-1} \mathbb{F}^{m}\left[\left[z^{-1}\right]\right]$. The set $z^{-1} \mathbb{F}^{m}\left[\left[z^{-1}\right]\right]$ is a vector

Received December $20^{\text {th }}, 2012$, Revised February $21^{\text {st }}, 2013$, Accepted for publication February $22^{\text {nd }}, 2013$. Copyright () 2013 Published by ITB Journal Publisher, ISSN: 2337-5760, DOI: 10.5614/j.math.fund.sci.2013.45.1.4 
space consisting of power series in $z^{-1}$ with coefficients in signal space $\mathbb{W}=\mathbb{F}^{m}$. These fenomena lead us to some questions: are both behaviors, according Willems [2] and Fuhrmann [3], equivalent? What is the relationship between these two concepts of behavior? This paper addresses part of these questions. Particularly, we show that for the case of a finite dimensional, time invariant, linear discrete system, behavior according to Willems is also behavior according to Fuhrmann.

\section{$2 \quad$ Preliminaries}

From now on, let $\mathbb{F}$ be an arbitrary field and $\mathbb{F}^{m}$ be the space of all $m$-tuples with components in $\mathbb{F}$. Notation $\mathbb{F}\left(\left(z^{-1}\right)\right)$ stands for the set of all formal series of the form $\sum_{i=n_{f}}^{-\infty} f_{i} z^{i}$ where $f_{i} \in \mathbb{F}$ and $n_{f} \in \mathbb{Z}$, that is, it can be written as

$$
\mathbb{F}\left(\left(z^{-1}\right)\right)=\left\{\sum_{i=n_{f}}^{-\infty} f_{i} z^{i} \mid f_{i} \in \mathbb{F}, n_{f} \in \mathbb{Z}\right\} .
$$

If $f(z)=\sum_{i=n_{f}}^{-\infty} f_{i} z^{i}$ and $g(z)=\sum_{i=n_{g}}^{-\infty} g_{i} z^{i}$ are both elements in $\mathbb{F}\left(\left(z^{-1}\right)\right)$, then the operations of addition and multiplication are defined by

$$
(f+g)(z)=\sum_{i=m a k s\left\{n_{f}, n_{g}\right\}}^{-\infty}\left(f_{i}+g_{i}\right) z^{i},
$$

and

$$
(f g)(z)=\sum_{k=n_{f}+n_{g}}^{-\infty} h_{k} z^{k},
$$

where $h_{k}=\sum_{i=-\infty}^{-\infty} f_{i} g_{k-i}$. Note that $h_{k}$ is well-defined, since it contains only a finite number of non-zero terms. The set $\mathbb{F}\left(\left(z^{-1}\right)\right)$ forms a field and is called the field of truncated Laurent series [4].

The set of all polynomials of the form $\sum_{i=0}^{n_{f}} f_{i} z^{i}$ where $f_{i} \in \mathbb{F}$ and $n_{f}$ is a nonnegative integer, is denoted by $\mathbb{F}[z]$. It can also be witten as 


$$
\mathbb{F}[z]=\left\{\sum_{i=0}^{n_{f}} f_{i} z^{i} \mid f_{i} \in \mathbb{F}, n_{f} \in \mathbb{N} \cup\{0\}\right\} .
$$

The set $\mathbb{F}[z]$ can be expressed as a subset of $\mathbb{F}\left(\left(z^{-1}\right)\right)$ as follows:

$$
\mathbb{F}[z]=\left\{\sum_{i=n f}^{0} f_{i} z^{-i} \mid f_{i} \in \mathbb{F}, n_{f} \in \mathbb{Z}-\mathbb{N}\right\} .
$$

The set $\mathbb{F}[z]$, coupled with the standard component-wise addition and multiplication, forms an Euclidean domain [4].

The set of all formal series having indeterminate $z^{-1}$ and coefficients in the field $\mathbb{F}$, is denoted by $\mathbb{F}\left[\left[z^{-1}\right]\right]$. This set can be expressed as follows:

$$
\mathbb{F}\left[\left[z^{-1}\right]\right]=\left\{\sum_{i=0}^{-\infty} f_{i} z^{i} \mid f_{i} \in \mathbb{F}, i=0,-1,-2, \ldots\right\} .
$$

Similar to the above polynomial ring, the set $\mathbb{F}\left[\left[z^{-1}\right]\right]$, coupled with standard addition and multiplication, forms an Euclidean Domain.

The field of truncated Laurent series can be decomposed by the polynomial domain part and formal series domain part as follows [4]:

$$
\mathbb{F}\left(\left(z^{-1}\right)\right)=\mathbb{F}[z] \oplus z^{-1} \mathbb{F}\left[\left[z^{-1}\right]\right]
$$

where

$$
z^{-1} \mathbb{F}\left[\left[z^{-1}\right]\right]=\left\{\sum_{i=-1}^{-\infty} f_{i} z^{-i} \mid f_{i} \in \mathbb{F}, i=-1,-2, \ldots\right\} .
$$

It is clear that the above definitions and notations, which are defined in terms of elements in the field $\mathbb{F}$, can be directly generalized in terms of elements in the vector space $\mathbb{F}^{m}$ and obtain the corresponding sets $\mathbb{F}^{m}\left(\left(z^{-1}\right)\right), \mathbb{F}^{m}[z]$, and $\mathbb{F}^{m}\left[\left[z^{-1}\right]\right]$. All of these sets can be formed as vector spaces over the field $\mathbb{F}$ and satisfy the following decomposition equation

$$
\mathbb{F}^{m}\left(\left(z^{-1}\right)\right)=\mathbb{F}^{m}[z] \oplus z^{-1} \mathbb{F}^{m}\left[\left[z^{-1}\right]\right] .
$$

Further, the set $\mathbb{F}^{m}[z]$ can also be identified by $\mathbb{F}[z]^{m}$, the set of $m$-tuples of elements in $\mathbb{F}[z]$ 


$$
\left(p_{i}(z)\right)=\left(\begin{array}{c}
p_{1}(z) \\
p_{2}(z) \\
\vdots \\
p_{m}(z)
\end{array}\right)
$$

Hence, the set $\mathbb{F}^{m}[z]$ can be considered as a module over the ring $\mathbb{F}[z]$. Similar identifications also work for $\mathbb{F}^{m}\left[\left[z^{-1}\right]\right]$ and $\mathbb{F}^{m}\left(\left(z^{-1}\right)\right)$.

The set $\mathbb{F}[z]^{m \times n}$ is defined as follows:

$$
\mathbb{F}[z]^{m \times n}=\left\{\left(a_{i j}(z)\right) \mid a_{i j} \in \mathbb{F}[z], 1 \leq i \leq m, 1 \leq j \leq n\right\} .
$$

Similar to the vector case, elements in $\mathbb{F}[z]^{m \times n}$ can be identified as elements in the set $\mathbb{F}^{m \times n}[z]$, that is

$$
\mathbb{F}^{m \times n}[z]=\left\{\sum_{i=0}^{n_{f}} f_{i} z^{i} \mid f_{i} \in \mathbb{F}^{m \times n}, n_{f} \in \mathbb{N} \cup\{0\}\right\} .
$$

The set $\mathbb{F}[z]^{m \times n}$ is a module over the ring $\mathbb{F}[z]$.

We close this section by introducing the term shift invariant subspace which will be one property of a behavior. First, following the decomposition (1), we have the projection operator on the space of formal series $z^{-1} \mathbb{F}^{m}\left[\left[z^{-1}\right]\right]$ along the polynomial space $\mathbb{F}^{m}[z]$ :

$$
\begin{array}{rlll}
\pi_{-}: \mathbb{F}^{m}\left(\left(z^{-1}\right)\right) & \rightarrow z^{-1} \mathbb{F}^{m}\left[\left[z^{-1}\right]\right] \\
\sum_{i=-\infty}^{n_{f}} f_{i} z^{i} & \mapsto \quad \sum_{i=-\infty}^{-1} f_{i} z^{i} .
\end{array}
$$

The shift operator $\sigma$ is, then, defined by

$$
\begin{aligned}
\sigma: \quad z^{-1} \mathbb{F}^{m}\left[\left[z^{-1}\right]\right] & \rightarrow z^{-1} \mathbb{F}^{m}\left[\left[z^{-1}\right]\right] \\
\sum_{i=1}^{\infty} h_{i} z^{-i} & \mapsto \pi_{-}\left(z \sum_{i=1}^{\infty} h_{i} z^{-i}\right),
\end{aligned}
$$


Definition 1. Let $\sigma$ be the shift operator defined in (2), and $\mathfrak{B}$ be a subspace of $z^{-1} \mathbb{F}^{m}\left[\left[z^{-1}\right]\right]$. The subspace $\mathfrak{B}$ is called shift invariant if $\sigma(\mathfrak{B}) \subseteq \mathfrak{B}$.

Note that having defined the two notions above, we can introduce an action from the domain $\mathbb{F}[z]$ to the space $z^{-1} \mathbb{F}^{m}\left[\left[z^{-1}\right]\right]$ which makes it become $\mathbb{F}[z]$ module. Particularly, the space $z^{-1} \mathbb{F}^{m}\left[\left[z^{-1}\right]\right]$ is an $\mathbb{F}[z]$-module with operation of multiplication defined as follows:

$$
z * h=\pi_{-} z h
$$

for all $z \in \mathbb{F}[z]$ and $h \in z^{-1} \mathbb{F}^{m}\left[\left[z^{-1}\right]\right]$.

\section{Behavior}

This section contains the main issue of this paper, that is the relevancy between the two ways of defining a behavior. The first subsection discusses the behavior by Willems's and Fuhrmann's point of view. The definition of the dynamic system will be discussed at the beginning of this section.

\subsection{Willems's Version of a Behavior}

This paper concentrates on finite dimensional, time invariant, discrete linear systems. Willems [2] defines a dynamic system as a triple, $\sum=(\mathbb{T}, \mathbb{W}, \mathfrak{B})$, where $\mathbb{T} \subseteq \mathbb{R}$ as time axis, $\mathbb{W}$ is called signal space, $\mathbb{W}^{\mathbb{T}}$ is the set of all transformations from $\mathbb{T}$ to $\mathbb{W}$, and $\mathfrak{B} \subseteq \mathbb{W}^{\mathbb{T}}$ is called the behavior. The behavior of a linear system with discrete time is defined by Willems [2] as the set of all trajectories of the dynamical system.

Consider a finite dimensional, time invariant, discrete linear system that satisfies the following difference equation:

$$
x_{k+1}=A x_{k}+B u_{k}
$$

where $x_{k} \in \mathbb{F}^{n}, u_{k} \in \mathbb{F}^{m}, k=0,1, \ldots$, for some $A$, an $n \times n$ matrix and $B$, an $n \times m$ matrix with entries in the field $\mathbb{F}$. Let $x_{0} \in \mathbb{F}^{n}$ and a sequence of inputs $u_{0}, u_{1}, \ldots$ be given, we obtain the corresponding state sequence $x_{1}, x_{2}, \ldots$ which satisfies Eq. (3). Let represent the state sequence in the form of a formal series 


$$
x\left(z^{-1}\right)=x_{1} z^{-1}+x_{2} z^{-2}+\ldots=\sum_{i=1}^{\infty} x_{i} z^{-i} .
$$

Then, the behavior in Willems's [2] context can be identified by

$$
\mathfrak{B}=\left\{\sum_{i=1}^{\infty} x_{i} z^{-i} \mid x_{i} \text { satisfy (3), for some } x_{0} \in \mathbb{F}^{n} \text { and } u_{i} \in \mathbb{F}^{m}, i=0,1,2, \ldots\right\} .
$$

Before discussing the definition of a behavior in Fuhrmann's point of view, we will first discuss the definition of a complete subspace of $z^{-1} \mathbb{F}^{m}\left[\left[z^{-1}\right]\right]$.

For each $n \in \mathbb{Z}_{+}$, let $P_{n}$ be the projection on $z^{-1} \mathbb{F}^{m}\left[\left[z^{-1}\right]\right]$ defined as

$$
\begin{aligned}
& P_{n}: \quad z^{-1} \mathbb{F}^{m}\left[\left[z^{-1}\right]\right] \quad \rightarrow z^{-1} \mathbb{F}^{m}\left[\left[z^{-1}\right]\right] \\
& \sum_{i=1}^{\infty} h_{i} z^{-i} \quad \mapsto \sum_{i=1}^{n} h_{i} z^{-i} .
\end{aligned}
$$

We say that a subset $\mathfrak{B} \subseteq z^{-1} \mathbb{F}^{m}\left[\left[z^{-1}\right]\right]$ is complete if the following statement is satisfied:

if for any $w=\sum_{i=1}^{\infty} w_{i} z^{-i} \in z^{-1} \mathbb{F}^{m}\left[\left[z^{-1}\right]\right], P_{N}(w) \in P_{N}(\mathfrak{B})$ for all $N$, then $w \in \mathfrak{B}$.

\subsection{Fuhrmann's Version of a Behavior}

In the previous subsection we already discussed Willems's [2] definition of a dynamical system as triple, $\sum=(\mathbb{T}, \mathbb{W}, \mathfrak{B})$, where $\mathbb{T} \subseteq \mathbb{R}$ is the times axis, $\mathbb{W}$ is called a signal space, $\mathbb{W}^{\mathbb{T}}$ is the set of all transformations from $\mathbb{T}$ to $\mathbb{W}$, and $\mathfrak{B} \subseteq \mathbb{W}^{\mathbb{T}}$ is called the behavior. Fuhrmann adopted this concept for discrete linear systems by restricting the time set $\mathbb{T}$ with the set of all natural numbers, denoted by $\mathbb{Z}_{+}$, and restricting $\mathbb{W}$ with $\mathbb{F}^{m}$, the colomn vector space over the field $\mathbb{F}$. However, Fuhrmann defines a behavior as a linear, shift invariant, and complete subspace of $z^{-1} \mathbb{F}^{m}\left[\left[z^{-1}\right]\right][3]$.

Even though Willems and Fuhrmann have a different point of view in defining a behavior, there is a linkage between both definitions of a behavior. That linkage is described in Theorem 3. 
Theorem 3. Let $\mathfrak{B}$ be the behavior according to Willems for time invariant, discrete linear system satisfying difference Eq. (3), that is,

$$
\mathfrak{B}=\left\{\sum_{i=1}^{\infty} x_{i} z^{-i} \mid x_{i} \text { satisfy (3), for some } x_{0} \in \mathbb{F}^{n} \text { and } u_{i} \in \mathbb{F}^{m}, i=0,1,2, \ldots\right\} \text {. }
$$

Then the set $\mathfrak{B}$ is a shift invariant and complete subspace of $z^{-1} \mathbb{F}^{m}\left[\left[z^{-1}\right]\right]$. In other words, $\mathfrak{B}$ is a behavior in Fuhrmann's [3] point of view.

\section{Proof. Let}

$$
\mathfrak{B}=\left\{\sum_{i=1}^{\infty} x_{i} z^{-i} \mid x_{i} \text { satisfy (3), for some } x_{0} \in \mathbb{F}^{n} \text { and } u_{i} \in \mathbb{F}^{m}, i=0,1,2, \ldots\right\}
$$

be a behavior for time invariant, discrete linear system. First of all, we will show that $\mathfrak{B}$ is a subspace of $z^{-1} \mathbb{F}^{m}\left[\left[z^{-1}\right]\right]$. That is, it should be pointed out that $\mathfrak{B}$ is a nonempty subset of $z^{-1} \mathbb{F}^{m}\left[\left[z^{-1}\right]\right]$ that is closed under operation of addition and scalar multiplication.

1. We will show that $\mathfrak{B}$ is non-empty, and that is so because

$$
0_{\mathfrak{B}}=0 . z^{-1}+0 . z^{-2}+\ldots+0 . z^{-n}+\ldots \in \mathfrak{B} .
$$

2. It is clear by definition that $\mathfrak{B}$ is a subset of $z^{-1} \mathbb{F}^{m}\left[\left[z^{-1}\right]\right]$.

3. We will show that $\mathfrak{B}$ is closed under addition. Let $x\left(z^{-1}\right), y\left(z^{-1}\right) \in \mathfrak{B}$, where $x\left(z^{-1}\right)=\sum_{i=1}^{\infty} x_{i} z^{-1}$ and $y\left(z^{-1}\right)=\sum_{i=1}^{\infty} y_{i} z^{-1}, x_{i}, y_{i}$ satisfying (3), for some $x_{0}, y_{0} \in \mathbb{F}^{n}$, and $u_{i}, v_{i} \in \mathbb{F}^{m}, i=0,1, \ldots$ We obtain

$$
x\left(z^{-1}\right)+y\left(z^{-1}\right)=\sum_{i=1}^{\infty} x_{i} z^{-1}+\sum_{i=1}^{\infty} y_{i} z^{-1}=\sum_{i=1}^{\infty}\left(x_{i}+y_{i}\right) z^{-1} .
$$

By assumption, $x_{i}$ and $y_{i}$ satisfy (3), that is

$$
x_{i}=A x_{i-1}+B u_{i-1} \text { and } y_{i}=A y_{i-1}+B v_{i-1} .
$$


Hence, $\quad x_{i}+y_{i}=A\left(x_{i-1}+y_{i-1}\right)+B\left(u_{i-1}+v_{i-1}\right)$ for some $x_{0}+y_{0} \in \mathbb{F}^{n}$, and $u_{i}+v_{i} \in \mathbb{F}^{m}$. Consequently, $x\left(z^{-1}\right)+y\left(z^{-1}\right) \in \mathfrak{B}$. Thus, it is proved that $\mathfrak{B}$ is closed under addition.

4. We shall show that $\mathfrak{B}$ is closed under scalar multiplication. Let $\alpha \in \mathbb{F}$ and $x\left(z^{-1}\right) \in \mathfrak{B}$, where $x\left(z^{-1}\right)=\sum_{i=1}^{\infty} x_{i} z^{-1}, x_{i}$ satisfy (3), for some $x_{0} \in \mathbb{F}^{n}$, and $u_{i} \in \mathbb{F}^{m}$, where $i=0,1,2, \ldots$. We have

$$
\alpha \cdot x\left(z^{-1}\right)=\alpha\left(\sum_{i=1}^{\infty} x_{i} z^{-1}\right)=\sum_{i=1}^{\infty}\left(\alpha x_{i}\right) z^{-1} .
$$

By assumption, $x_{i}$ satisfy (3), that is $x_{i}=A x_{i-1}+B u_{i-1}$. Consequently

$$
\alpha x_{i}=A\left(\alpha x_{i-1}\right)+B\left(\alpha u_{i-1}\right)
$$

for some $\alpha x_{0} \in \mathbb{F}^{n}$, and $\alpha u_{i} \in \mathbb{F}^{m}$. So, $\alpha x\left(z^{-1}\right) \in \mathfrak{B}$.

Therefore, since $\mathfrak{B}$ is a subset of $z^{-1} \mathbb{F}^{m}\left[\left[z^{-1}\right]\right]$ that is non-empty and closed under addition and scalar multiplication, we can conclude that $\mathfrak{B}$ is a subspace.

Now, we shall show that $\mathfrak{B}$ is shift invariant. In other words, we have to show that $\sigma(\mathfrak{B}) \subseteq \mathfrak{B}$, where $\sigma$ is the shift operator defined according to (2). Let $g \in \sigma(\mathfrak{B})$. There exists

$$
x\left(z^{-1}\right)=\sum_{i=1}^{\infty} x_{i} z^{-i} \in \mathfrak{B}
$$

where $x_{i}$ satisfy (3), for some $x_{0} \in \mathbb{F}^{n}$, and $u_{i} \in \mathbb{F}^{m}$, so $g=\sigma\left(x\left(z^{-1}\right)\right)=\pi_{-} z x\left(z^{-1}\right)$. We have

$$
\begin{aligned}
\pi_{-} z x\left(z^{-1}\right) & =\pi_{-} z\left(\sum_{i=1}^{\infty} x_{i} z^{-i}\right) \\
& =\pi_{-} z\left(\sum_{i=1}^{\infty}\left(A x_{i-1}+B u_{i-1}\right) z^{-i}\right)
\end{aligned}
$$




$$
\begin{aligned}
& =\pi_{-} \sum_{i=1}^{\infty}\left(A x_{i-1}+B u_{i-1}\right) z^{-i+1} \\
& =\sum_{i=2}^{\infty}\left(A x_{i-1}+B u_{i-1}\right) z^{-i+1} .
\end{aligned}
$$

Thus $\pi_{-} z x\left(z^{-1}\right) \in \mathfrak{B}$ implies $\sigma(\mathfrak{B}) \subseteq \mathfrak{B}$. Hence $\mathfrak{B}$ is shift invariant.

Finally, we will show that $\mathfrak{B}$ is complete. From (3) we have:

$$
\begin{aligned}
& x_{1}=A x_{0}+B u_{0}=\left(\begin{array}{ll}
A & B
\end{array}\right)\left(\begin{array}{l}
x_{0} \\
u_{0}
\end{array}\right) \\
& x_{2}=A x_{1}+B u_{1}=A^{2} x_{0}+A B u_{0}+B u_{1}=\left(\begin{array}{lll}
A^{2} & A B & B
\end{array}\right)\left(\begin{array}{l}
x_{0} \\
u_{0} \\
u_{1}
\end{array}\right) \\
& \vdots \\
& x_{k}=A x_{k-1}+B u_{k-1}=A^{k} x_{0}+\sum_{i=0}^{k-1} A^{k-1-i} B u_{i} \\
& =\left(\begin{array}{llllll}
A^{k} & A^{k-1} B & A^{k-2} B & \ldots & A B & B
\end{array}\right)\left(\begin{array}{c}
x_{0} \\
u_{0} \\
u_{1} \\
u_{2} \\
\vdots \\
u_{k-1}
\end{array}\right) .
\end{aligned}
$$

Let $f=\sum_{i=1}^{\infty} f_{i} z^{-i} \in z^{-1} \mathbb{F}^{m}\left[\left[z^{-1}\right]\right]$ and for any $n \in\{1,2, \ldots\}$ we have

$$
P_{n}(f)=\sum_{i=1}^{n} f_{i} z^{-i} \in P_{n}(\mathfrak{B}) .
$$

We obtain $f \in \mathfrak{B}$ if we can show that there exists $y_{0} \in \mathbb{F}^{m}$ and for all $n \in\{1,2, \ldots\}$, we can determine $v_{n-1} \in \mathbb{F}^{p}$ such that 


$$
f_{i}=\left(\begin{array}{llllll}
A^{i} & A^{i-1} B & A^{i-2} B & \ldots & A B & B
\end{array}\right)\left(\begin{array}{c}
y_{0} \\
v_{0} \\
v_{1} \\
v_{2} \\
\vdots \\
v_{i-1}
\end{array}\right) \text {, for } i=1,2, \ldots, n .
$$

This last expected fact will be shown using mathematical induction on the natural numbers $n$. For $n=1$, we have $f_{1} z^{-1}=P_{1}(f) \in P_{1}(\mathfrak{B})$. That means there exists $w^{1} \in \mathfrak{B}$ such that $f_{1} z^{-1}=P_{1}\left(w^{1}\right)$. Write $w^{1}=\sum_{i=1}^{\infty} w_{i}^{1} z^{-i} \in \mathfrak{B}$. This means that there exists $x_{0}^{1} \in \mathbb{F}^{m}$ and $u_{0}^{1}, u_{1}^{1}, \ldots \in \mathbb{F}^{p}$ such that

$$
w_{i}^{1}=\left(\begin{array}{llllll}
A^{i} & A^{i-1} B & A^{i-2} B & \ldots & A B & B
\end{array}\right)\left(\begin{array}{c}
x_{0}^{1} \\
u_{0}^{1} \\
u_{1}^{1} \\
u_{2}^{1} \\
\vdots \\
u_{i-1}^{1}
\end{array}\right) \text {, for all } i=1,2, \ldots
$$

Particularly $f_{1}=w_{1}^{1}=\left(\begin{array}{ll}A & B\end{array}\right)\left(\begin{array}{c}x_{0}^{1} \\ u_{0}^{1}\end{array}\right)$. Write $y_{0}=x_{0}^{1}, v_{0}=u_{0}^{1}$. Therefore, there exists $y_{0}=x_{0}^{1} \in \mathbb{F}^{m}$ and for $n=1$ there exists $v_{0}=u_{0}^{1} \in \mathbb{F}^{p}$ such that

$$
f_{1}=w_{1}^{1}=\left(\begin{array}{ll}
A & B
\end{array}\right)\left(\begin{array}{c}
x_{0}^{1} \\
u_{0}^{1}
\end{array}\right) .
$$

Thus, the expected fact is true for $n=1$.

Assume that the expected fact is true for $1 \leq n \leq k$. That is, we assume we already obtained

$$
y_{0} \in F^{m} \quad \text { and } \quad v_{1}, v_{2}, \ldots, v_{k-1} \in \mathbb{F}^{p}
$$

such that 


$$
f_{i}=\left(\begin{array}{llllll}
A^{i} & A^{i-1} B & A^{i-2} B & \ldots & A B & B
\end{array}\right)\left(\begin{array}{c}
y_{0} \\
v_{0} \\
v_{1}^{1} \\
v_{2}^{1} \\
\vdots \\
v_{i-1}
\end{array}\right)
$$

for $i=1,2, \ldots, k$.

We will determine $v_{k}$ such that the expected fact holds for $n=k+1$. Consider that

$$
\sum_{i=1}^{k+1} f_{i} z^{-i}=P_{k+1}(f) \in P_{k+1}(\mathfrak{B})
$$

Suppose $w^{k+1} \in \mathfrak{B}$ such that $\sum_{i=1}^{k+1} f_{i} z^{-i}=P_{k+1}(f)=P_{k+1}\left(w^{k+1}\right)$. Let

$$
x_{0}^{k+1} \in \mathbb{F}^{m}, u_{0}^{k+1}, u_{1}^{k+1}, \ldots \in \mathbb{F}^{p}
$$

such that $w^{k+1}=\sum_{j=1}^{\infty} w_{j}^{k+1} z^{-j}$ where

$$
w_{j}^{k+1}=\left(\begin{array}{llllll}
A^{j} & A^{j-1} B & A^{j-2} B & \ldots & A B & B
\end{array}\right)\left(\begin{array}{c}
x_{0}^{k+1} \\
u_{0}^{k+1} \\
u_{1}^{k+1} \\
u_{2}^{k+1} \\
\vdots \\
u_{j-1}^{k+1}
\end{array}\right)
$$

for all $j=1,2, \ldots$ Thus, we have

$$
f_{1}=w_{1}^{k+1} ; f_{2}=w_{2}^{k+1} ; \ldots ; f_{k}=w_{k}^{k+1} ; f_{k+1}=w_{k+1}^{k+1} .
$$

That is, for any $i=1,2, \ldots, k$ we have 


$$
\begin{aligned}
f_{i}=\left(\begin{array}{llllll}
A^{i} & A^{i-1} B & A^{i-2} B & \ldots & A B & B
\end{array}\right)\left(\begin{array}{c}
y_{0} \\
v_{0} \\
v_{1} \\
v_{2} \\
\vdots \\
v_{i-1}
\end{array}\right) \\
=\left(\begin{array}{llllll}
A^{i} & A^{i-1} B & A^{i-2} B & \ldots & A B & B
\end{array}\right)\left(\begin{array}{c}
x_{0}^{k+1} \\
u_{0}^{k+1} \\
u_{1}^{k+1} \\
u_{2}^{k+1} \\
\vdots \\
u_{i-1}^{k+1}
\end{array}\right) .
\end{aligned}
$$

\section{Particularly}

$$
\begin{aligned}
f_{k}=\left(\begin{array}{llllll}
A^{k} & A^{k-1} B & A^{k-2} B & \ldots & A B & B
\end{array}\right)\left(\begin{array}{c}
y_{0} \\
v_{0} \\
v_{1} \\
v_{2} \\
\vdots \\
v_{k-1}
\end{array}\right) \\
=\left(\begin{array}{llllll}
A^{k} & A^{k-1} B & A^{k-2} B & \ldots & A B & B
\end{array}\right)\left(\begin{array}{c}
x_{0}^{k+1} \\
u_{0}^{k+1} \\
u_{1}^{k+1} \\
u_{2}^{k+1} \\
\vdots \\
u_{k-1}^{k+1}
\end{array}\right) .
\end{aligned}
$$


Meanwhile we have

$$
\begin{aligned}
f_{k+1} & =\left(\begin{array}{llllll}
A^{k+1} & A^{k} B & A^{k-1} B & \ldots & A B & B
\end{array}\right)\left(\begin{array}{c}
x_{0}^{k+1} \\
u_{0}^{k+1} \\
u_{1}^{k+1} \\
u_{2}^{k+1} \\
\vdots \\
u_{k}^{k+1}
\end{array}\right) . \\
& =A f_{k}+B u_{k}^{k+1} .
\end{aligned}
$$

Hence, substituting the Eq. (5), we obtain

$$
f_{k+1}=A\left(\begin{array}{llllll}
A^{k} & A^{k-1} B & A^{k-2} B & \ldots & A B & B
\end{array}\right)\left(\begin{array}{c}
y_{0}^{k+1} \\
v_{0}^{k+1} \\
v_{1}^{k+1} \\
v_{2}^{k+1} \\
\vdots \\
v_{k-1}^{k+1}
\end{array}\right)+B u_{k}^{k+1} .
$$

So, by defining $v_{k}=u_{k}^{k+1}$, we have

$$
f_{k+1}=\left(\begin{array}{llllll}
A^{k+1} & A^{k} B & A^{k-1} B & \ldots & A B & B
\end{array}\right)\left(\begin{array}{c}
y_{0} \\
v_{0} \\
v_{1} \\
\vdots \\
v_{k}
\end{array}\right)
$$

That is, we obtain $v_{k}=u_{k}^{k+1}$ such that the expected fact holds for $n=k+1$. Consequently, $f \in \mathfrak{B}$. Thus $\mathfrak{B}$ is complete.

As a conclusion, we have proved that $\mathfrak{B}$ is a shift invariant and complete subspace of $z^{-1} \mathbb{F}^{m}\left[\left[z^{-1}\right]\right]$. In other words, $\mathfrak{B}$ is a behavior in Fuhrmann's point of view. 


\section{Conclusion}

Willems $[1,2]$ defined a dynamical system as triple, $\sum=(\mathbb{T}, \mathbb{W}, \mathfrak{B})$, where $\mathbb{T} \subseteq \mathbb{R}$ is the time axis, $\mathbb{W}$ is signal space, and $\mathfrak{B} \subseteq \mathbb{W}^{\mathbb{T}}$ is the behavior and $\mathbb{W}^{\mathbb{T}}$ is the set of all transformations from $\mathbb{T}$ to $\mathbb{W}$. The behavior of a linear system, based on Willems's [2] point of view, is defined as the set of all trajectories of the dynamical system. Meanwhile, Fuhrmann [3] defined the behavior of a discrete time, linear systems as a shift invariant and complete subspace of the corresponding formal series module $z^{-1} \mathbb{F}^{m}\left[\left[z^{-1}\right]\right]$. In this article we have shown that the behavior of a system in Willems's point of view [2], for the case of a finite dimensional, time invariant, discrete linear system, is also behavior according to Fuhrmann [3]. The converse of this result is expected to be true and is still an open question under investigation.

\section{Acknowledgments}

This work is supported by Program Riset dan Inovasi KK ITB 2012 according to Surat Perjanjian Pelaksanaan Riset No.: 425/I.1.C01/PL/2012 signed on February $1^{\text {st }}, 2012$. The authors would like to thank to the reviewers for their valuable comments and suggestions.

\section{References}

[1] Willems, J.C., The Behavioral Approach to Open and Interconnected Systems, IEEE Control Systems Magazine, 27(6), pp. 46-99, 2007.

[2] Willems, J.C., From Time Series to Linear Systems. Part I: FiniteDimensional Linear Time Invariant Systems, Automatica, 22(5), pp. 561580, 1986.

[3] Fuhrmann, P.A., A Study of Behavior, Linear Algebra and Its Applications, 351-352, pp. 303-380, 2002.

[4] Fuhrmann, P.A., Linear Systems and Operators in Hilbert Space, McGraw-Hill, New York, 19813. 